\title{
A Generalization of Archimedes' Theorem on the Area of a Parabolic Segment
}

\section{Armen GRIGORYAN, Szymon IGNACIUK and Maciej PAROL}

\begin{abstract}
Archimedes' well known theorem on the area of a parabolic segment says that this area is $4 / 3$ of the area of a certain inscribed triangle. In this paper we generalize this theorem to the $n$-dimensional euclidean space, $n \geq 3$. It appears that the ratio of the volume of an $n$ dimensional solid bounded by an $(n-1)$-dimensional hyper-paraboloid and an $(n-1)$-dimensional hyperplane and the volume of a certain inscribed cone (we analogously repeat Archimedes' procedure) depends only on the dimension of the euclidean space and it equals to $2 n /(n+1)$.
\end{abstract}

\section{Introduction}

Let us recall Archimedes' (287-212 BC) theorem on the area of a parabolic segment. Let $\mathrm{P}$ be a parabola in the euclidean plane $\mathbb{R}^{2}$ and let $A B$ be a chord of $\mathrm{P}$. Denote by $C^{\prime}$ the midpoint of $A B$. Consider the line $\ell$ which passes through $C^{\prime}$ and is parallel to the symmetry line of $\mathrm{P}$. Denote by $C$ the intersection of $\ell$ with the parabola $\mathrm{P}$, see Figure 1 .

The point $C$ is often called the center of the arc $A B$ of the parabola P. Denote by $\mathrm{S}$ the parabolic segment bounded by the parabola $\mathrm{P}$ and the chord $A B$. Archimedes' famous result says that

$$
\operatorname{Area}(\mathrm{S})=\frac{4}{3} \cdot \operatorname{Area}(\triangle A B C) .
$$

Key Words: Archimedes' theorem, parabolic segment, multiple integral, spherical coordinates

2010 Mathematics Subject Classification: 26B15, 28A75, 51M25.

Received: 24.09.2020

Accepted: 15.11 .2020 


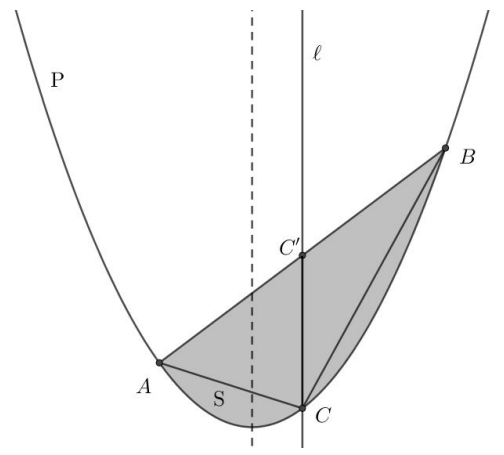

Figure 1: Classic version of Archimedes' theorem.

It is amazing that this relationship between the area of the parabolic segment $\mathrm{S}$ and the area of the triangle $\triangle A B C$ does not depend on the shape of the parabola $\mathrm{P}$ or the chord $A B$. Of course Archimedes' proof does not involve the integral calculus. In fact he gave two proofs of the above theorem. One of them involves Eudoxus' (408-355 BC) method of exhaustion and another one is based on moving center of masses of certain arcs. For learning Archimedes' original considerations we refer to [3, p. 251], see also [2].

In this paper we generalize Archimedes' theorem to the $n$-dimensional euclidean space $\mathbb{R}^{n}$. Let us start with some notations. We denote by $\mathbb{N}$ the set of all positive integers. Let $n \in \mathbb{N}, n \geq 3$. For $a_{1}, \ldots, a_{n-1} \in(0 ;+\infty)$ we set

$$
\mathrm{P}_{n-1}:=\left\{\left(x_{1} \ldots, x_{n}\right) \in \mathbb{R}^{n}: x_{n}=\sum_{k=1}^{n-1} a_{k} x_{k}^{2}\right\} .
$$

Notice that $\mathrm{P}_{n-1}$ is an $(n-1)$-dimensional elliptic hyper-paraboloid. For $b_{1}, \ldots, b_{n} \in \mathbb{R}$ we consider an $(n-1)$-dimensional hyperplane $\mathrm{H}_{n-1}$ given by

$$
\mathrm{H}_{n-1}:=\left\{\left(x_{1} \ldots, x_{n}\right) \in \mathbb{R}^{n}: x_{n}=\sum_{k=1}^{n-1} b_{k} x_{k}+b_{n}\right\} .
$$

Obviously, $\mathrm{H}_{n-1}$ is not perpendicular to the hyperplane $\left\{\left(x_{1}, \ldots, x_{n-1}, 0\right): x_{i} \in\right.$ $\mathbb{R}, i=1, \ldots, n-1\}$. Notice that $\left(x_{1}, \ldots, x_{n}\right) \in \mathrm{P}_{n-1} \cap \mathrm{H}_{n-1}$ if and only if

$$
x_{n}=\sum_{k=1}^{n-1} a_{k} x_{k}^{2}=\sum_{k=1}^{n-1} b_{k} x_{k}+b_{n},
$$


which is equivalent to

$$
\left(x_{1}, \ldots, x_{n}\right) \in \mathrm{H}_{n-1} \quad \text { and } \quad \sum_{k=1}^{n-1}\left(\sqrt{a_{k}} x_{k}-\frac{b_{k}}{2 \sqrt{a_{k}}}\right)^{2}=b_{n}+\sum_{k=1}^{n-1} \frac{b_{k}^{2}}{4 a_{k}} .
$$

This means that the intersection $\mathrm{P}_{n-1} \cap \mathrm{H}_{n-1}$ contains more than one point if and only if

$$
b_{n}+\sum_{k=1}^{n-1} \frac{b_{k}^{2}}{4 a_{k}}>0
$$

Moreover, if the condition (0.4) is satisfied, then the number

$$
R:=\sqrt{b_{n}+\sum_{k=1}^{n-1} \frac{b_{k}^{2}}{4 a_{k}}}
$$

is well defined and the set

$$
\mathrm{E}_{n-2}:=\mathrm{P}_{n-1} \cap \mathrm{H}_{n-1}=\left\{\left(x_{1}, \ldots, x_{n}\right) \in \mathrm{H}_{n-1}: \sum_{k=1}^{n-1} \frac{\left(x_{k}-b_{k} /\left(2 a_{k}\right)\right)^{2}}{\left(R / \sqrt{a_{k}}\right)^{2}}=1\right\}
$$

is an $(n-2)$-dimensional ellipsoid. In what follows we assume that the condition (0.4) is satisfied. We denote by $c$ the center of the ellipsoid $\mathrm{E}_{n-2}$, i.e.

$$
c=\left(\frac{b_{1}}{2 a_{1}}, \frac{b_{2}}{2 a_{2}}, \ldots, \frac{b_{n-1}}{2 a_{n-1}}, \sum_{k=1}^{n-1} \frac{b_{k}^{2}}{2 a_{k}}+b_{n}\right),
$$

and we denote by $p(c)$ the intersection of the line

$$
\ell_{1}(c):=\left\{\left(\frac{b_{1}}{2 a_{1}}, \frac{b_{2}}{2 a_{2}}, \ldots, \frac{b_{n-1}}{2 a_{n-1}}, t\right): t \in \mathbb{R}\right\}
$$

with the hyper-paraboloid $\mathrm{P}_{n-1}$. We get

$$
p(c)=\mathrm{P}_{n-1} \cap \ell_{1}(c)=\left(\frac{b_{1}}{2 a_{1}}, \frac{b_{2}}{2 a_{2}}, \ldots, \frac{b_{n-1}}{2 a_{n-1}}, \sum_{k=1}^{n-1} \frac{b_{k}^{2}}{4 a_{k}}\right) .
$$

Finally we denote by $\mathrm{C}_{n}$ the convex hull of the union of the point $p(c)$ and the ellipsoid $\mathrm{E}_{n-2}$, i.e.

$$
\mathrm{C}_{n}=\operatorname{conv}\left(\mathrm{E}_{n-2} \cup p(c)\right):=\left\{\lambda x+(1-\lambda) y: \lambda \in[0 ; 1], x, y \in \mathrm{E}_{n-2} \cup p(c)\right\} .
$$




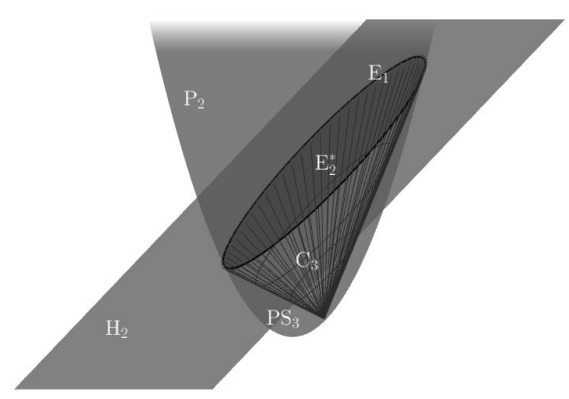

Figure 2: Three dimensional version of Archimedes' theorem.

Notice that $\mathrm{C}_{n}$ is an $n$-dimensional cone with the vertex at $p(c)$, see Figure 2 for the 3-dimensional case.

In what follows, we refer to $\mathrm{C}_{n}$ as a cone, for short. Comparing to the 2-dimensional case, the role of the parabolic segment plays the set

$$
\mathrm{PS}_{n}:=\left\{\left(x_{1} \ldots, x_{n}\right) \in \mathbb{R}^{n}: \sum_{k=1}^{n-1} a_{k} x_{k}^{2} \leq x_{n} \leq \sum_{k=1}^{n-1} b_{k} x_{k}+b_{n}\right\}
$$

which is an $n$-dimensional solid body bounded by the hyper-paraboloid $\mathrm{P}_{n-1}$ and the hyperplane $\mathrm{H}_{n-1}$. The role of the triangle $\triangle A B C$ plays the cone $\mathrm{C}_{n}$. It appears that the ratio of the volume of the hyper-parabolic segment $\mathrm{PS}_{n}$ and the volume of the cone $\mathrm{C}_{n}$ depends only on the dimension of the euclidean space, i.e. depends only on $n$. More precisely we prove the following result:

$$
\operatorname{Vol}_{n}\left(\mathrm{PS}_{n}\right)=\frac{2 n}{n+1} \cdot \operatorname{Vol}_{n}\left(\mathrm{C}_{n}\right)
$$

In particular,

$$
\mathrm{Vol}_{3}\left(\mathrm{PS}_{3}\right)=\frac{3}{2} \cdot \mathrm{Vol}_{3}\left(\mathrm{C}_{3}\right)
$$

Throughout this paper we write $\operatorname{Vol}_{n}(E)$ for the $n$-dimensional Lebesgue measure of a Lebesgue measurable set $E$, although it would be sufficient to operate on Jordan measurable sets and the Jordan measure.

\section{Main results}

We will compute multiple integrals by changing variables into spherical $n$ dimensional coordinates, see [1]. Additionally we will scale along the axes and translate appropriate transformation, in order to integrate comfortably over 
convex hulls of ellipsoids. The Jacobian (see [4, 234]) of such a transformation is basically well-known, so a technical Lemma 1.2 is not new.

Let $u$ and $v$ be non-negative integers. In what follows we formally replace the product $\prod_{i=u}^{v}$ by 1 if $v<u$, regardless what is the expression under the product symbol. We denote by $\mathbb{N}_{u, v}$ the set of all integers $k$ such that $u \leq k \leq v$, if $u \leq v$. We set $\mathbb{N}_{u, v}:=\emptyset$, if $u>v$.

Let $m \in \mathbb{N}, m \geq 2, \theta_{1}, \ldots, \theta_{m-1} \in \mathbb{R}$ and $\theta_{m}:=0\left(\right.$ so $\left.\cos \left(\theta_{m}\right)=1\right)$. Define the matrix $\mathbf{M}_{m}: \mathbb{N}_{1, m} \times \mathbb{N}_{1, m} \rightarrow \mathbb{R}$ by

$$
\mathbf{M}_{m}(p, q):= \begin{cases}\cos \left(\theta_{p}\right) \prod_{j=1}^{p-1} \sin \left(\theta_{j}\right), & \text { if } p \in \mathbb{N}_{1, m} \text { and } q=1, \\ \cos \left(\theta_{p}\right) \cos \left(\theta_{q-1}\right) \prod_{j=1}^{p-1} \sin \left(\theta_{j}\right), & \text { if } p \in \mathbb{N}_{2, m} \text { and } q \in \mathbb{N}_{2, p}, \\ -\prod_{j=1}^{p} \sin \left(\theta_{j}\right), & \text { if } p \in \mathbb{N}_{1, m-1} \text { and } q=p+1, \\ 0, & \text { if } p \in \mathbb{N}_{1, m-2} \text { and } q \in \mathbb{N}_{p+2, m} .\end{cases}
$$

For the convenience of the Reader we give an array form of the matrix $\mathrm{M}_{m}$ :

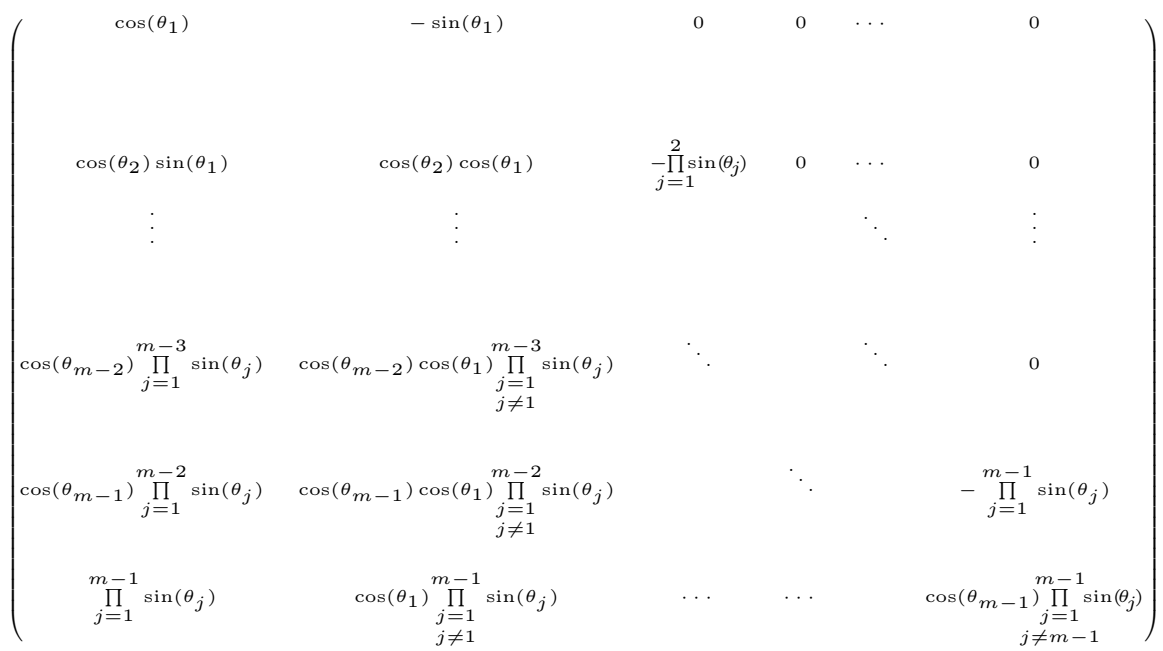

Lemma 1.1. The determinant of $\mathbf{M}_{m}$ satisfies the following equality

$$
\operatorname{det}\left(\mathbf{M}_{m}\right)=\prod_{k=1}^{m-2} \sin ^{m-k-1}\left(\theta_{k}\right)
$$


Proof. Obviously, $\operatorname{det}\left(\mathbf{M}_{2}\right)=1$. Assume $m \in \mathbb{N}, m \geq 3$. Using Laplace's expansion along the $m$ th column of the matrix $\mathbf{M}_{n}$, we get

$$
\begin{aligned}
\operatorname{det}\left(\mathbf{M}_{m}\right) & =(-1)^{2 m-1}\left(-\prod_{j=1}^{m-1} \sin \left(\theta_{j}\right)\right) \sin \left(\theta_{m-1}\right) \operatorname{det}\left(\mathbf{M}_{m-1}\right) \\
& +(-1)^{2 m}\left(\cos \left(\theta_{m-1}\right) \prod_{\substack{j=1 \\
j \neq m-1}}^{m-1} \sin \left(\theta_{j}\right)\right) \cos \left(\theta_{m-1}\right) \operatorname{det}\left(\mathbf{M}_{m-1}\right) \\
& =\prod_{j=1}^{m-2} \sin \left(\theta_{j}\right) \operatorname{det}\left(\mathbf{M}_{m-1}\right)
\end{aligned}
$$

By the mathematical induction, we get the desired formula.

Let $\alpha:=\left(\alpha_{1}, \ldots, \alpha_{m}\right) \in \mathbb{R}^{m}, \beta:=\left(\beta_{1}, \ldots, \beta_{m}\right) \in \mathbb{R}^{m}$. For each $k \in \mathbb{N}_{1, m}$ we set

$$
\varphi_{k}^{\left(\alpha_{k}, \beta_{k}\right)}\left(r, \theta_{1}, \theta_{2}, \ldots, \theta_{m-1}\right):=\alpha_{k} r \cos \left(\theta_{k}\right) \prod_{j=1}^{k-1} \sin \left(\theta_{j}\right)+\beta_{k}
$$

where $\left(r, \theta_{1}, \theta_{2}, \ldots, \theta_{m-1}\right) \in \mathbb{R}^{m}$. Recall that $\theta_{m}=0$, so $\cos \left(\theta_{m}\right)=1$. We define the mapping $\Phi_{m}^{(\alpha, \beta)}: \mathbb{R}^{m} \rightarrow \mathbb{R}^{m}$ by

$$
\Phi_{m}^{(\alpha, \beta)}:=\left(\varphi_{1}^{\left(\alpha_{1}, \beta_{1}\right)}, \ldots, \varphi_{m}^{\left(\alpha_{m}, \beta_{m}\right)}\right)
$$

So $\varphi_{k}^{\left(\alpha_{k}, \beta_{k}\right)}$ is the $k$ th coordinate function of $\Phi_{m}^{(\alpha, \beta)}$. The vector $\alpha$ controls a scaling along the axes while the vector $\beta$ controls a translation. Of course, the mapping $\Phi_{m}^{(\alpha, \beta)}$ has continuous partial derivatives of all orders.

Lemma 1.2. The Jacobian $\mathrm{J}_{\Phi_{m}^{(\alpha, \beta)}}$ of the mapping $\Phi_{m}^{(\alpha, \beta)}: \mathbb{R}^{m} \rightarrow \mathbb{R}^{m}$ satisfies the equality

$$
\mathrm{J}_{\Phi_{m}^{(\alpha, \beta)}}\left(r, \theta_{1}, \theta_{2}, \ldots, \theta_{m-1}\right)=r^{m-1}\left(\prod_{k=1}^{m} \alpha_{k}\right) \prod_{k=1}^{m-2} \sin ^{m-k-1}\left(\theta_{k}\right),
$$

for all $\left(r, \theta_{1}, \theta_{2}, \ldots, \theta_{m-1}\right) \in \mathbb{R}^{m}$. 
Proof. For $k \in \mathbb{N}_{1, m}$ we have

$$
\begin{aligned}
& \frac{\partial \varphi_{k}^{(\alpha, \beta)}}{\partial r}\left(r, \theta_{1}, \theta_{2}, \ldots, \theta_{m-1}\right)=\alpha_{k} \cos \left(\theta_{k}\right) \prod_{j=1}^{k-1} \sin \left(\theta_{j}\right), \\
& \frac{\partial \varphi_{k}^{(\alpha, \beta)}}{\partial \theta_{i}}\left(r, \theta_{1}, \theta_{2}, \ldots, \theta_{m-1}\right)= \begin{cases}r \alpha_{k} \cos \left(\theta_{k}\right) \cos \left(\theta_{i}\right) \prod_{\substack{j=1 \\
j \neq i}}^{k-1} \sin \left(\theta_{j}\right), \text { if } i \in \mathbb{N}_{1, k-1}, \\
-r \alpha_{k} \prod_{j=1}^{k} \sin \left(\theta_{j}\right), & \text { if } i=k, \\
0, & \text { if } i \in \mathbb{N}_{k+1, m-1} .\end{cases}
\end{aligned}
$$

Thus, by Lemma 1.1, we get

$$
\begin{aligned}
\mathrm{J}_{\Phi_{m}^{(\alpha, \beta)}}\left(r, \theta_{1}, \theta_{2}, \ldots, \theta_{m-1}\right) & =r^{m-1}\left(\prod_{k=1}^{m} \alpha_{k}\right) \operatorname{det}\left(\mathbf{M}_{m}\right) \\
& =r^{m-1}\left(\prod_{k=1}^{m} \alpha_{k}\right) \prod_{k=1}^{m-2} \sin ^{m-k-1}\left(\theta_{k}\right) .
\end{aligned}
$$

Remark 1.3. Set $1:=(1, \ldots, 1) \in \mathbb{R}^{m}$ and $\mathbf{0}:=(0, \ldots, 0) \in \mathbb{R}^{m}$. Then the mapping $\Phi_{m}^{(\mathbf{1}, \mathbf{0})}: \mathbb{R}^{m} \rightarrow \mathbb{R}^{m}$ restricted to the set $[0,+\infty) \times \mathbb{R}^{m-1}$ is a transformation of the classical spherical $m$-dimensional coordinates onto the Cartesian coordinates. In this case

$$
\mathrm{J}_{\Phi_{m}^{(1,0)}}\left(r, \theta_{1}, \theta_{2}, \ldots, \theta_{m-1}\right)=r^{m-1} \prod_{k=1}^{m-2} \sin ^{m-k-1}\left(\theta_{k}\right),
$$

for all $\left(r, \theta_{1}, \theta_{2}, \ldots, \theta_{m-1}\right) \in \mathbb{R}^{m}$ (compare this with the formula given in [1]).

Let $r>0$. Notice, that the mapping $\Phi_{m}^{(\mathbf{1 , 0})}$ transforms in a non-injective manner the closed $m$-orthotope $[0 ; r] \times[0 ; \pi]^{m-2} \times[0 ; 2 \pi]$ onto the closed ball $\mathbb{B}_{m}(r):=\left\{x \in \mathbb{R}^{m}:\|x\| \leq r\right\}$ of the radius $r$ (here \|\| means the euclidean norm). However, the restriction of the mapping $\Phi_{m}^{(\mathbf{1}, \mathbf{0})}$ to the open $m$-orthotope

$$
\Omega_{m}(r):=(0, r) \times(0 ; \pi)^{m-2} \times(0 ; 2 \pi)
$$

is a diffeomorphism. Moreover

$$
\Phi_{m}^{(\mathbf{1}, \mathbf{0})}\left(\Omega_{m}(r)\right) \subset \mathbb{B}_{m}(r) \quad \text { and } \quad \operatorname{Vol}_{m}\left(\Phi_{m}^{(\mathbf{1}, \mathbf{0})}\left(\Omega_{m}(r)\right)\right)=\operatorname{Vol}_{m}\left(\mathbb{B}_{m}(r)\right) .
$$


Theorem 1.4. Let $a_{1}, \ldots, a_{n-1} \in(0 ;+\infty), b_{1}, \ldots, b_{n} \in \mathbb{R}$, and let the condition (0.4) be satisfied. Then

$$
\operatorname{Vol}_{n}\left(\mathrm{PS}_{n}\right)=\frac{2 n}{n+1} \cdot \operatorname{Vol}_{n}\left(\mathrm{C}_{n}\right)
$$

where $\mathrm{C}_{n}$ and $\mathrm{PS}_{n}$ are defined in (0.7) and (0.8), respectively.

Proof. Let us define $\mathrm{E}_{n-1}^{*}:=\operatorname{conv}\left(\mathrm{E}_{n-2}\right)$. Denote by $\mathrm{D}_{n-1}$ the projection of $\mathrm{E}_{n-1}^{*}$ onto the hyperplane $\left\{\left(x_{1}, \ldots, x_{n-1}, 0\right): x_{i} \in \mathbb{R}, i=1, \ldots, n-1\right\}$. By (0.6), we have

$$
\mathrm{D}_{n-1}=\left\{\left(x_{1}, \ldots, x_{n-1}, 0\right) \in \mathbb{R}^{n}: \sum_{k=1}^{n-1}\left[\sqrt{a_{k}} \cdot\left(x_{k}-\frac{b_{k}}{2 a_{k}}\right)\right]^{2} \leq R^{2}\right\},
$$

where $R$ is defined as in (0.5). Put $m:=n-1$,

$$
\alpha:=\left(\frac{1}{\sqrt{a_{1}}}, \ldots, \frac{1}{\sqrt{a_{n-1}}}\right) \quad \text { and } \quad \beta:=\left(\frac{b_{1}}{2 a_{1}}, \ldots, \frac{b_{n-1}}{2 a_{n-1}}\right),
$$

and consider the mapping $\Phi_{n-1}^{(\alpha, \beta)}: \mathbb{R}^{n-1} \rightarrow \mathbb{R}^{n-1}$ defined by (1.1). The restriction of $\Phi_{n-1}^{(\alpha, \beta)}$ to the open $(n-1)$-orthotope $\Omega_{n-1}(R)$, defined by $(1.2)$, is a diffeomorphism. Moreover,

$$
\Phi_{n-1}^{(\alpha, \beta)}\left(\Omega_{n-1}(R)\right) \subset D_{n-1} \text { and } \operatorname{Vol}_{n-1}\left(\Phi_{n-1}^{(\alpha, \beta)}\left(\Omega_{n-1}(R)\right)\right)=\operatorname{Vol}_{n-1}\left(D_{n-1}\right) .
$$

Hence, by the transformation formula (see $[4,252])$ and by Lemma 1.2, we get

$$
\begin{aligned}
& \operatorname{Vol}_{n}\left(\mathrm{PS}_{n}\right)=\overbrace{\iint_{\mathrm{D}_{n-1}} \ldots \int}^{n-1}\left(R^{2}-\sum_{k=1}^{n-1} a_{k}\left(x_{k}-\frac{b_{k}}{2 a_{k}}\right)^{2}\right) \mathrm{d} x_{1} \mathrm{~d} x_{2} \ldots \mathrm{d} x_{n-1}
\end{aligned}
$$

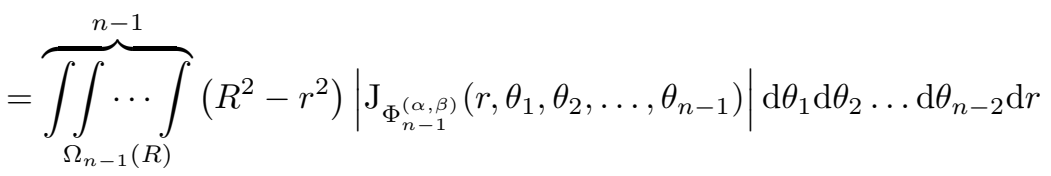

$$
\begin{aligned}
& =\int_{0}^{R} \int_{0}^{2 \pi} \underbrace{\int_{0}^{\pi} \ldots \int_{0}^{\pi}}_{n-3}\left(R^{2}-r^{2}\right)\left|r^{n-2}\left(\prod_{k=1}^{n-1} \frac{1}{\sqrt{a_{k}}}\right) \prod_{k=1}^{n-3} \sin ^{n-k-2}\left(\theta_{k}\right)\right| \mathrm{d} \theta_{1} \mathrm{~d} \theta_{2} \ldots \mathrm{d} \theta_{n-2} \mathrm{~d} r
\end{aligned}
$$




$$
\begin{aligned}
& =2^{n-1} \int_{0}^{R} \underbrace{\int_{0}^{\frac{\pi}{2}} \ldots \int_{0}^{\frac{\pi}{2}}}_{n-2}\left(R^{2} r^{n-2}-r^{n}\right)\left(\prod_{k=1}^{n-1} \frac{1}{\sqrt{a_{k}}}\right) \prod_{k=1}^{n-3} \sin ^{n-k-2}\left(\theta_{k}\right) \mathrm{d} \theta_{1} \mathrm{~d} \theta_{2} \ldots \mathrm{d} \theta_{n-2} \mathrm{~d} r \\
& =2^{n-1} \cdot\left(\frac{R^{n+1}}{n-1}-\frac{R^{n+1}}{n+1}\right) \cdot\left(\prod_{k=1}^{n-1} \frac{1}{\sqrt{a_{k}}}\right) \cdot \frac{\pi}{2} \cdot \prod_{k=1}^{n-3} \int_{0}^{\frac{\pi}{2}} \sin ^{n-k-2}\left(\theta_{k}\right) \mathrm{d} \theta_{k} \\
& =\frac{2^{n-1} \pi R^{n+1}}{n^{2}-1} \cdot\left(\prod_{k=1}^{n-1} \frac{1}{\sqrt{a_{k}}}\right) \cdot \prod_{k=1}^{n-3} \frac{\sqrt{\pi} \Gamma\left(\frac{n-k-1}{2}\right)}{2 \Gamma\left(\frac{n-k}{2}\right)} \\
& =\frac{4 \pi^{\frac{n-1}{2}} R^{n+1}}{\left(n^{2}-1\right) \Gamma\left(\frac{n-1}{2}\right)} \prod_{k=1}^{n-1} \frac{1}{\sqrt{a_{k}}} \text {. }
\end{aligned}
$$

If $n=3$ then $\underbrace{\int_{0}^{\pi} \cdots \int_{0}^{\pi}}_{n-3}$ just disappear and the result remains valid.

Now we determine $\operatorname{Vol}_{n}\left(\mathrm{C}_{n}\right)$. Since $\mathrm{C}_{n}$ is an $n$-dimensional cone with base $\mathrm{E}_{n-1}^{*}$ and vertex at $p(c)$. Therefore

$$
\operatorname{Vol}_{n}\left(\mathrm{C}_{n}\right)=\frac{1}{n} \operatorname{dist}\left(p(c) ; \mathrm{H}_{n-1}\right) \cdot \operatorname{Vol}_{n-1}\left(\mathrm{E}_{n-1}^{*}\right),
$$

where $\operatorname{dist}\left(p(c) ; \mathrm{H}_{n-1}\right)$ denotes the euclidean distance between $p(c)$ and $\mathrm{H}_{n-1}$. Since

$$
\left(\operatorname{dist}\left(p(c) ; \mathrm{H}_{n-1}\right)\right)^{2}=\frac{\left(\sum_{k=1}^{n-1} \frac{b_{k}^{2}}{2 a_{k}}-\sum_{k=1}^{n-1} \frac{b_{k}^{2}}{4 a_{k}}+b_{n}\right)^{2}}{\sum_{k=1}^{n-1} b_{k}^{2}+1},
$$

SO

$$
\operatorname{dist}\left(p(c) ; \mathrm{H}_{n-1}\right)=R^{2}\left(\sum_{k=1}^{n-1} b_{k}^{2}+1\right)^{-\frac{1}{2}}
$$

Furthermore,

$$
\operatorname{Vol}_{n-1}\left(\mathrm{E}_{n-1}^{*}\right)=\overbrace{\iint_{\mathrm{D}_{n-1}} \ldots \int}^{n-1} \sqrt{\sum_{k=1}^{n-1} b_{k}^{2}+1} \mathrm{~d} x_{1} \mathrm{~d} x_{2} \ldots \mathrm{d} x_{n-1} .
$$


Proceeding as above, we get

$$
\begin{aligned}
& \operatorname{Vol}_{n-1}\left(\mathrm{E}_{n-1}^{*}\right)=
\end{aligned}
$$

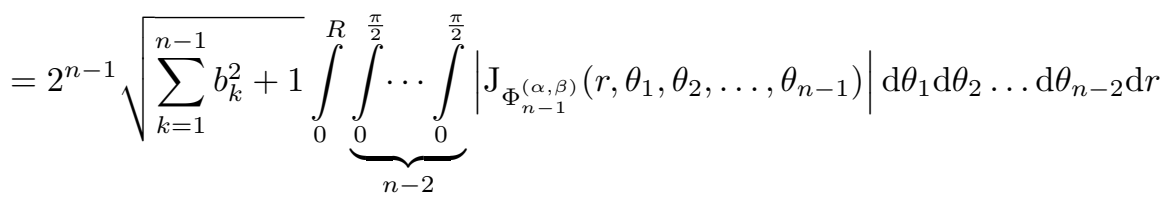

$$
\begin{aligned}
& =2^{n-1} \sqrt{\sum_{k=1}^{n-1} b_{k}^{2}+1} \cdot \frac{R^{n-1}}{n-1} \cdot\left(\prod_{k=1}^{n-1} \frac{1}{\sqrt{a_{k}}}\right) \cdot \frac{\pi}{2} \cdot \prod_{k=1}^{n-3} \int_{0}^{\frac{\pi}{2}} \sin ^{n-k-2}\left(\theta_{k}\right) \mathrm{d} \theta_{k} \\
& =\frac{2 \pi^{\frac{n-1}{2}} R^{n-1}}{(n-1) \Gamma\left(\frac{n-1}{2}\right)} \sqrt{\sum_{k=1}^{n-1} b_{k}^{2}+1} \prod_{k=1}^{n-1} \frac{1}{\sqrt{a_{k}}} \text {. }
\end{aligned}
$$

Hence, by (1.3) and (1.4), we have

$$
\operatorname{Vol}_{n}\left(\mathrm{C}_{n}\right)=\frac{2 \pi^{\frac{n-1}{2}} R^{n+1}}{n(n-1) \Gamma\left(\frac{n-1}{2}\right)} \prod_{k=1}^{n-1} \frac{1}{\sqrt{a_{k}}}
$$

Finally, we get

$$
\frac{\operatorname{Vol}_{n}\left(\mathrm{PS}_{n}\right)}{\operatorname{Vol}_{n}\left(\mathrm{C}_{n}\right)}=\frac{2 n}{n+1}
$$

Corollary 1.5. In the 3-dimensional case (see Figure 2) the ratio of the volume of the parabolic segment $\mathrm{PS}_{3}$ and the volume of the cone $\mathrm{C}_{3}$ is equal to $3 / 2$.

\section{References}

[1] L. E. Blumenson, A derivation of n-Dimensional Spherical Coordinates, The American Mathematical Monthly, Vol. 67, No. 1 (1960), pp. 63-66.

[2] T. Dance, G. Swain, Archimedes' Quadrature of Parabola Revisited, Mathematics Magazine, Vol. 71, No. 2 (1998), pp. 123-130.

[3] T. L. Heath, The Works of Archimedes, C. J. Clay and Sons, London, 1897.

[4] W. Rudin, Principles of the Mathematical Analysis, third edition, McGraw-Hill, Inc., New York, 1976. 
Armen GRIGORYAN,

Department of Mathematical Analysis

The John Paul II Catholic University of Lublin

ul. Konstantynów 1 H, 20-708 Lublin, Poland.

ORCID: 0000-0001-6775-3953

Email: armen@kul.lublin.pl

Szymon IGNACIUK,

Department of Applied Mathematics and Computer Science

University of Life Sciences in Lublin

ul. Głęboka 28, 20-612 Lublin, Poland.

ORCID: 0000-0001-8288-2833

Email: szymon.ignaciuk@up.lublin.pl

Maciej PAROL,

Department of Mathematical Analysis

The John Paul II Catholic University of Lublin

ul. Konstantynów 1 H, 20-708 Lublin, Poland.

ORCID: 0000-0001-8981-2491

Email: mparol@kul.lublin.pl 